\title{
Carnets
}

Revue électronique d'études françaises de l'APEF

Deuxième série - 15 | 2019

Jules Verne et les pouvoirs de l'imagination

\section{Un carrefour des Voyages extraordinaires}

La pièce Voyage à travers l'impossible (1882)

\section{Volker Dehs}

\section{(2) OpenEdition}

Journals

Édition électronique

URL : http://journals.openedition.org/carnets/9069

DOI : $10.4000 /$ carnets.9069

ISSN : 1646-7698

Éditeur

APEF

Référence électronique

Volker Dehs, «Un carrefour des Voyages extraordinaires », Carnets [En ligne], Deuxième série - 15 | 2019, mis en ligne le 31 janvier 2019, consulté le 02 mai 2019. URL : http://journals.openedition.org/ carnets/9069; DOl : 10.4000/carnets.9069

Ce document a été généré automatiquement le 2 mai 2019.

\section{(c) (†) (S)}

Carnets est mis à disposition selon les termes de la licence Creative Commons - Atribution - Pas d'utilisation commerciale 4.0 International. 


\section{Un carrefour des Voyages extraordinaires}

La pièce Voyage à travers l'impossible (1882)

Volker Dehs

\section{NOTE DE L'AUTEUR}

Directeur de publication du Bulletin de la Société Jules Verne (Paris). Membre du comité de rédaction de la revue Verniana-Études Jules Verne.

\section{Une rétrospective littéraire depuis longtemps enfantée}

1 Après dix années d'un labeur incessant, consacré selon le projet de l'éditeur Hetzel à "résumer toutes les connaissances géographiques, géologiques, physiques, astronomiques, amassées par la science moderne, et de refaire (...) l'histoire de l'univers » (Hetzel, 1867 : II ), Jules Verne exprima pour la première fois l'intention de développer une vision rétrospective sur son œuvre. Ainsi, le journaliste Adrien Marx rapporta en 1875 :

Jules Verne prépare les Merveilles de la science, "grande machine », comme on dit maintenant, qui empruntera sa mise en scène non seulement à la peinture, au velours, à la soie et au corps de ballet, mais encore aux agents dynamiques de la physique, de la chimie et de la mécanique (Margot, 2004 : 46).

Mais la réalisation d'une autre pièce de théâtre, l'adaptation du roman Le Tour du monde en quatre-vingts jours, renvoya la réalisation de ce projet à plus tard. Deux années après, en août 1875 , le romancier revint à son idée et donna quelques précisions à son éditeur :

Il y a quelques mois, je vous ai dit que je comptais faire ce livre-là ${ }^{1}$, qu'il serait le résumé des Voyages extraordinaires, que j'embarquerais dans un appareil plus lourd que l'air tous nos bonshommes, Fergusson, Aronnax, Fogg, Clawbonny, etc. et que je les ferais naviguer au-dessus de tout notre monde. Je ne renonce point à cette idée ( Correspondance II, $2001: 52$ ). 
3 Projet encyclopédique oblige, on trouve une première manifestation de cette intention synthétisante dans le roman L'T̂le mystérieuse, publié à la même époque (1874-75) et que Daniel Compère a qualifié, avec raison, de « roman carrefour » (Compère, 1991 : 117). En effet, ce roman n'est pas seulement une "prolonge» de deux romans précédents (Les Enfants du capitaine Grant et Vingt mille lieues sous les mers) par la reprise de certains personnages, il réunit aussi des éléments et motifs constitutifs des romans déjà publiés et anticipe sur des aspects des romans à venir. Ce procédé composite correspond au caractère microcosmique du roman: l'espace formé par une île réunit - contre toute logique scientifique - des aspects contrastifs et même opposés de la nature, donne le cadre temporel dans lequel est décrit le développement de la civilisation humaine, de sa création jusqu'à sa destruction finale. En même temps, le caractère autoréférentiel du roman est contrebalancé par une dimension intertextuelle, car Jules Verne se réfère au genre de la robinsonnade, non seulement en refaisant le modèle de Robinson Crusoe de Defoe, évoqué dans le texte par plusieurs allusions, mais implicitement aussi au roman Le Cratère de Fenimore Cooper, dont il reprend le dénouement. Ces mêmes principes créateurs président à ce que doit devenir la pièce Voyage à travers l'impossible.

\section{Les conditions d'une collaboration difficile}

4 Jules Verne avait prévu tout d'abord d'écrire sans collaboration une pièce classique, puis privilégié le genre du roman pour réaliser son projet rétrospectif, c'est finalement le mélodrame qui l'emporta. Comme dans le cas des adaptations précédentes (Le Tour du monde (1874), Les Enfants du capitaine Grant (1878) et Michel Strogoff de 1880) il dut accepter la collaboration d'Adolphe d'Ennery, qui lui fut imposée par les directeurs de théâtre. Depuis les années 1830, d'Ennery (1811-1899) passait pour le « maître du mélodrame » et le calcul des directeurs qui appréciaient Verne comme romancier, mais se fiaient moins à son talent dramatique, devait s'avérer juste - du moins pour deux des pièces citées qui furent jouées au plus grand théâtre de Paris jusqu'en 1940.

5 La collaboration entre Verne et d'Ennery était difficile, d'autant plus que le romancier détestait le caractère vulgaire de la maîtresse de son collaborateur, dont il devait supporter la présence pendant de longs séjours passés à leur domicile au cap d'Antibes. Il est d'autant plus surprenant que Verne servit de témoin à d'Ennery lors de son mariage avec cette même Clémence Desgranges en mai $1881 .$. Ce qui est connu de la correspondance entre les deux auteurs ${ }^{2}$ montre que les séjours à Antibes servaient à fixer en commun le plan des pièces dont Jules Verne seul élabora au fur et à mesure le texte corrigé ensuite par d'Ennery plus expérimenté. Cette constatation vaut également pour la pièce Voyage à travers l'impossible qui fut commencée en 1877 mais terminée seulement au printemps 1882 - au grand mécontentement des éditeurs de Verne, lesquels n'appréciaient pas le sujet qui leur paraissait trop sortir du cadre des Voyages extraordinaires. Ainsi, Hetzel fils écrivit à son père le 11 février 1880 :

Verne est féru de son idée de «À travers l'impossible». C'est lui qui l'a donnée à

d'Ennery et non d'Ennery à lui. C'est une insanité et quand même le livre serait un

chef-d'œuvre le titre serait seul un coup porté à son genre de production. Il doit

faire de l'« extraordinaire » et non de l' « impossible » mais il n'y a pas à espérer de

faire revenir ce Breton de ses idées préconçues (Correspondance III, 2002 : 79).

6 Jules Verne poursuivait cette collaboration, car les résultats lui assuraient une carrière dramatique qu'il avait toujours ambitionnée dès ses débuts littéraires et lui permettait de 
concilier cette ambition avec les activités d'un romancier, qu'il n'avait continuées qu'avec hésitation depuis 1863, sur les insistances d'Hetzel. Les rapports avec d'Ennery se détérioraient toutefois à partir de 1883 parce que Verne avait osé d'adapter cette fois seul son roman Kéraban-le-têtu (qui fut un échec retentissant) :

Ce qui est certain également c'est que d'Ennery ne fut pas content d'avoir été abandonné, et que sa femme Gisette fit tout ce qu'elle put pour démolir la pièce de Jules Verne. J'ai entendu ma mère dire à ce sujet: Ce que peut la haine d'une femme ! » (Larochelle, $1960: 165)^{3}$

7 La brouille définitive se produit seulement en 1890, après deux tentatives infructueuses d'adapter en commun le roman de Verne, Les Tribulations d'un Chinois en Chine (1879) (Dehs, 2011a). À partir de cette époque, ce furent d'autres dramaturges que le romancier autorisa à adapter sa prose (Dehs, 2011b).

\section{Dépasser l'extraordinaire par l'impossible... et son échec}

8 Le Voyage à travers l'impossible, "drame fantastique » en trois actes et vingt tableaux, fut créé le 25 novembre au Théâtre de la Porte Saint-Martin, un établissement comportant 1.800 places, qui était spécialisé dans les fééries et pièces à grand spectacle. Quatre-vingtdix-sept représentations s'ensuivirent jusqu'en mars 1883, mais le public avait déjà commencé à bouder le spectacle. Les recettes - dont d'Ennery pouvait encaisser $7 \%$ et Verne $5 \%$, soit 19.335,99 francs pour ce dernier ${ }^{4}$ - suffisaient de peu à couvrir les frais énormes de l'opulente mise en scène. Par la suite, le directeur Paul Clèves abandonna l'idée de transporter le drame au théâtre du Châtelet (qui comprenait le double de places) comme on l'avait fait après les succès retentissants du Tour du monde et de Michel Strogoff. On ignore jusqu'à présent si des représentations en Angleterre et aux États-Unis ont eu lieu, car en septembre 1882 d'Ennery et Verne avaient reçu chacun la somme de 3.500 francs pour la vente de la pièce à leur agent anglais, Marcus L. Mayer ${ }^{5}$. La pièce elle-même avait été destinée à dépasser les splendeurs des deux drames précédents, à réaliser sur la scène du jamais vu, ni dans la réalité ni dans les Voyages extraordinaires. Peut-être le public, averti depuis longtemps par de nombreux articles dans les journaux, avait-il de trop grandes espérances et dut-il constater que la nouvelle pièce ne dépassait et n'atteignait même pas la force dramatique du Tour du monde et de Michel Strogoff. Toujours est-il que, par cette ambition de surenchérir sur les succès précédents, la pièce réalise précisément ce que son intrigue critique : l'ambition et la démesure humaines !

Aucune reprise du vivant des auteurs n'est connue, et comme la pièce ne fut pas publiée et qu'aucun manuscrit ne se trouvait dans les archives des héritiers, le texte du Voyage à travers l'impossible passait pour perdu ${ }^{6} . .$. jusqu'à ce que le publiciste Gilbert Sigaux retrouvât une copie établie en 1882 pour le bureau de la Censure du ministère de l'Intérieur ${ }^{7}$. C'est ce texte qui fut publié pour la première fois par François Raymond et Robert Pourvoyeur en 1981 et, dans une version complétée d'un tableau, par Agnès Marcetteau-Paul et Jean-Michel Margot en 2005 (Verne, 1981 et 2005).

La redécouverte de cette pièce avait été accueillie avec enthousiasme par les spécialistes qui s'apercevaient que, bien avant la publication du roman posthume Paris au $\mathrm{XX}^{e}$ siècle (1994), le cliché d'un Jules Verne, chantre inconditionnel du progrès scientifique, n'était désormais plus recevable. Mais quelles sont les raisons de cette faillite impressionnante 
auprès de son public? Si les critiques de l'époque étaient unanimes à vanter le décor éblouissant du spectacle, la qualité des ballets (accompagnés de la musique considérée comme médiocre du compositeur Oscar Lagoanère) et le jeu des artistes, tous expérimentés ou voués à une carrière prometteuse, ils éreintaient avec une belle unanimité la pièce elle-même et son caractère réactionnaire du point de vue religieux. Comme ils trouvaient cet aspect incompatible avec l'esprit perçu comme optimiste et positif des Voyages extraordinaire, la plupart d'entre eux l'attribuaient tout droit à d'Ennery - à tort, comme nous le montrent la correspondance et les témoignages des éditeurs Hetzel. Le critique dramatique Edmond Stoullig en fournit un bon exemple dans son compte rendu du National :

M. d'Ennery a entrepris de nous faire assister, pour la millième fois, à la lutte du bon ange contre le mauvais ange, mais ce que Scribe n'a pas mis dans Robert le Diable , ce que Dumas n'a pas mis dans Don Juan de Marana, ce que Goethe n'a pas mis dans Faust, le génie mélodramatique de M. Dennery l'a insinué dans ce voyage bizarre d'un archange qui est un organiste et qui s'appelle Volsius: je veux parler d'un mysticisme catholico-réactionnaire qui essaie de faire pleurer de l'eau bénite au public.

À part cette lubie religioso-frondeuse qui marque une nouvelle manière chez $\mathrm{M}$. d'Ennery, - espérons que ce sera la dernière, ô mon Dieu ! - il n'y a pas le plus petit grain d'originalité et de fantaisie dans ce voyage, où l'on coudoie pourtant les types les plus originaux de Jules Verne: le docteur Ox, le capitaine Nemo et le fils d'Hatteras (Stoullig, 1882).

Stoullig se serait abstenu de son attribution trop facile s'il avait su que Jules Verne, des deux auteurs, était le catholique pratiquant ; d'Ennery, lui, était de religion juive !

\section{Une intrigue déconcertante}

12 L'action du Voyage à travers l'impossible débute et se termine au Danemark, à une époque non déterminée du $\mathrm{XIX}^{\mathrm{e}}$, dans le château d'Andernak qui est la résidence de madame de Traventhal et de sa petite-fille Ève ainsi que du fiancé de celle-ci, Georges Hatteras. Il sert de cadre au voyage des protagonistes, en trois étapes successives : le centre de la Terre (l'origine de la création), les profondeurs de la mer avec l'Atlantide (le passé de l'humanité) et la planète lointaine d'Altor (représentation de l'avenir de la Terre). Ces étapes reprennent par leurs destinations ainsi que par leurs personnages chacune une œuvre célèbre de Jules Verne : Voyage au centre de la Terre (1864), Vingt mille lieues sous les mers (1869/70) ainsi que les romans lunaires, De la Terre à la Lune (1865) et Autour de la Lune (1870). À cette tripartition correspondent trois couples qui constituent les personnages principaux de la pièce. Ils entreprennent ce voyage à travers le temps et l'espace grâce à une liqueur mystérieuse qui transporte les protagonistes « avec la rapidité de la foudre et dans les conditions d'une vie nouvelle jusqu'aux milieux interdits à l'homme » (Verne, 2005 : 34). Les auteurs renoncent donc à toute vraisemblance scientifique, réclamée par Hetzel, et qui distingue l'œuvre romanesque de Jules Verne, tout en caractérisant l'entreprise décrite comme une transgression non seulement spatiale et temporelle, mais explicitement morale.

Premier couple : les fiancés Éva et Georges qui est le personnage principal de l'œuvre et qui est dévoré par l'obsession de réaliser les explorations déjà entreprises par les héros des Voyages extraordinaires, mais qui n'avaient pas pu arriver jusqu'au but espéré. Bien sûr, 
l'amour pour sa fiancée en souffre, à tel point que ses proches craignent pour son esprit exalté :

Ma chère Éva, je t'aime, tu le sais, mon cœur est à toi, il est à vous, ma mère, mais mon imagination est plus forte que lui ! À chaque heure de la nuit ou du jour, elle m'emporte loin de ce château, loin de ce pays, au-delà des limites terrestres, presque dans les mondes inconnus, et j'entends une voix qui me crie : plus avant, plus loin, plus loin encore ! (Ibid. : 28).

La fiancée, quant à elle, partage l'espoir exprimé par sa grand-mère que Georges, par leurs soins réunis, « comprendra que le bonheur est ici, dans la vie de famille, et Dieu fera le reste » (ibid. : 22). Éva veut calmer Georges par l'influence de la foi et le détourner de ses projets de voyage. Mais les deux femmes se trompent fort, surtout Mme de Traventhal qui a mandé l'assistance du docteur Ox, célèbre médecin (qui provient de la nouvelle de Verne, Une fantaisie du docteur Ox de 1872, reprise dans le recueil Le Docteur Ox de 1874 ; recueil auquel on reviendra).

Survient alors le deuxième couple formé par les représentants du Bon et du Mal. Ox, sous le manteau d'un savant renommé, matérialiste, athée, et même quelque peu démoniaque, poursuit en réalité des objectifs très personnels. Comme il s'est épris d'Éva qu'il veut conquérir par son pouvoir et l'angoisse qu'il répand, il essaie de se défaire de Georges. Loin de le guérir, il veut au contraire l'inciter au voyage et le rendre fou. Il lui révèle qu'il est en fait le fils du capitaine John Hatteras (le conquérant du pôle Nord, mort fou, dont Verne avait décrit l'expédition dans son roman Voyages et aventures du capitaine Hatteras, de 1866), et Georges lui fait aveuglément confiance. Heureusement, Ox trouve un rival puissant dans le personnage de l'organiste Volsius (qui n'a pas de modèle dans les Voyages extraordinaires, mais qui incarnera des héros de Jules Verne, le professeur Lidenbrock, le capitaine Nemo et Michel Ardan). Volsius s'allie à Éva et, réunissant en sa personne la foi et la musique, l'art le plus parfait, il essaie de détourner Georges de sa démesure qui, selon lui, ne le mènera qu'à la ruine morale :

Mais ouvrez les saints livres et vous y trouverez de plus ambitieux orgueils, de plus audacieuses rébellions, de plus redoutables châtiments! Et ceux-là bien réels, et ceux-là si terribles que le docteur Ox lui-même redouterait de les affronter. (...) Je vais essayer de vous montrer dans quels profonds abîmes s'engloutit un sacrilège orgueil (Ibid. : 40).

Le troisième couple remplit une fonction purement humoristique et s'oppose au caractère austère des deux premiers, élément contrastif que Jules Verne s'efforçait d'introduire dans toutes ses œuvres dramatiques et en prose. Le premier est le maitre de danse un peu enfantin Tartelet, engagé par madame de Traventhal et directement sorti du roman L'École des Robinson (1882) où, sous le nom de T. Artelett, il joue le même rôle, dans une situation analogue : il sert de compagnon au capricieux Godfrey Morgan qui, avant de consentir au mariage avec sa fiancée Phina, veut entreprendre de lointains voyages. Les deux hommes partent en mer et échouent après un naufrage sur une île inhabitée où ils font de leur mieux pour jouer "au Robinson ». Finalement, tout est un leurre mis en scène par l'oncle de Godfrey qui, par une aventure arrangée, a voulu ramener son neveu à la raison en le laissant courir des dangers simulés. Toutefois, cet oncle a été trompé à son tour par un rival acharné, ce qui fait que la plupart des dangers ont été bien réels ! - Au cours du Voyage à travers l'impossible, ce Tartelet se lie d'amitié avec le touriste danois Axel Valdemar, simpliste lui aussi (mais sans précédent immédiat dans les romans verniens, sauf son prénom qui renvoie au narrateur du Voyage au centre de la Terre, tandis que son patronyme reprend le nom de plusieurs rois danois plutôt que de rappeler le titre d'une 
nouvelle de Poe). Si l'humour de leurs dialogues paraît au lecteur de nos jours bien désuet, il semble avoir été apprécié par le public d'autrefois. Toutefois, par son obsession de chercher un diamant pour faire de l'effet à sa fiancée, Valdemar parodie en même temps la traque furtive d'Ox à l'égard d'Éva et la quête de l'absolu menée par Georges. Le cours du voyage démontrera d'ailleurs non seulement la vanité du savoir, mais aussi la relativité des richesses humaines.

Grâce à la liqueur mirifique du docteur Ox, on pénètre bientôt par le Vésuve dans les entrailles de la Terre. Celle-ci avait expulsé les héros du roman vernien alors qu'ils n'avaient réalisé qu'un $40^{\circ}$ de leur trajet. Georges est bien décidé à atteindre le centre du globe :

Ox : Allons, Georges Hatteras, plus avant, plus avant encore!

Georges : Je vous suis, docteur ! C'est l'abîme... et l'abîme attire et j'irai jusqu'à ses dernières profondeurs!

Volsius: La contemplation de ces merveilles ne suffira-t-elle pas à satisfaire votre ambition de voyageur? (...)

Georges : À quoi servirait la nouvelle puissance vitale donnée à notre corps par le docteur $0 x$, s'il ne s'agissait que d'aller où d'autres sont allés devant nous, où vous êtes allé vous-même? Ici est l'extraordinaire et non pas l'impossible ! (Ibid. : 60-61)

Volsius, sous les traits du professeur Lidenbrock, n'est plus le savant hargneux du roman, mais argumente en artiste qui sait savourer la nature par la contemplation, terme qui implique chez Verne toujours une signification religieuse et s'oppose au matérialisme analytique du savant ${ }^{8}$. La supériorité de l'artiste se révèle de nouveau lorsque Éva est enlevée par des troglodytes préhistoriques et trop facilement déclarée perdue par $0 \mathrm{x}$, alors que Volsius la sauve en envoûtant les "primitifs » par son jeu au violon. Georges pousse « plus avant » et atteint avec ses compagnons le feu central où des phénix, follets et salamandres forment un ballet. Le tabou du point sublime est profané et réduit à (ou transformé en) un spectacle esthétique.

Poursuivant dans le $2^{\mathrm{e}}$ acte un prétendu monstre marin, les voyageurs se retrouvent sur la plate-forme du sous-marin Nautilus, commandé par le capitaine Nemo (incarné une fois de plus par Volsius). Au grand étonnement d'Ox, Nemo se révèle «fervent croyant » et prétend que l'athéisme est « né de l'orgueil ou de la crainte » (ibid.: 95). Il se livre alors à un monologue contre les dérives de la civilisation qui fut considéré inopportun par la Censure de la III ${ }^{\mathrm{e}}$ République, qui le raya dans le manuscrit. On ignore si le texte complet ne fut jamais joué ; ce qui est toutefois attesté par des journalistes, c'est que l'acteur Joumard, pendant les répétitions, joua le rôle du capitaine Nemo sous les traits de Jules Verne, astuce significative qui fut abandonnée à la première.

Arrivés au fond des océans, les voyageurs assistent à une résurrection du passé par la découverte de l'Atlantide animée. Georges y devient roi et, enivré par la gloire, abandonne Éva pour épouser une princesse, mais se ravise et repart à la conquête du ciel.

Ce n'est pas vers la Lune qu'on repart dans le $\mathrm{III}^{\mathrm{e}}$ acte, mais vers la planète lointaine Altor (du latin, «nourrisseur ») car Georges, de plus en plus exalté, veut « aller se perdre dans l'infini » (ibid.: 141) : «En sorte qu'après être remonté vers le passé, vers l'Atlantide, nous allons nous jeter à travers l'avenir » (ibid.: 148). La capitale est tout entière bâtie en or incrusté de pierres précieuses, ce qui dévalorise complètement le diamant que Valdemar avait trouvé au centre de la Terre ${ }^{9}$. La population a fait tant de progrès que «tout le monde est devenu savant ", raconte un Altorien (Volsius déguisé). " Les cordonniers font des vers et les boulangers de l'astronomie, nous manquons d'ouvriers et nous serons 
forcés d'en venir à décréter l'ignorance obligatoire » (ibid.: 162). Cette dernière remarque doit avoir particulièrement déplu à Hetzel et aux républicains progressistes car elle tourne en dérision un débat en cours au parlement français visant à étendre l'instruction obligatoire aux jeunes filles, projet vivement combattu par les conservateurs et le clergé. naturelles, à l'encontre des conseils de Volsius, mais encouragé par Ox, Georges Hatteras s'investit dans un projet surhumain pour «satisfaire aux besoins d'une civilisation et d'une industrie à outrance » (ibid. : 164). Alors que l'orgueil d'Ox est blessé et même brisé parce qu'Éva l'a définitivement éconduit en lui déclarant sa haine, Georges veut introduire un océan à l'intérieur de la planète. "Chantez, buvez, dansez ", crie-t-il, sombrant dans la mégalomanie, aux Altoriens, « car vous avez accompli une œuvre sans pareille, c'est un glorieux triomphe de l'homme sur la nature, c'est un magnifique spectacle, que l'on payerait de sa vie » (ibid. : 173).

L'œuvre gigantesque s'accomplit et provoque une terrible explosion. La planète s'écroule, seuls les deux principes du Bon et du Mal subsistent: "Tous les personnages sont renversés et semblent morts. Ox et Volsius seuls sont demeurés debout et se regardent d'un air de défi » (ibid. : 175).

Tous se retrouvent au château d'Andernak et on ne sait pas clairement si on avait rêvé ou vécu la réalité. Ce qui est sûr cependant, c'est que Georges a perdu la raison. Volsius propose alors à Ox d'unir leurs efforts pour le sauver :

Vous êtes une puissante incarnation de cette science pour qui le corps est tout, et qui ne croit en rien dans l'avenir. Je suis, moi, l'humble serviteur de la foi, et je compte pour rien cette humble enveloppe terrestre. Rendez la vie à ce corps, je m'efforcerai, moi, de réveiller sa raison et de rendre le calme et la force à son âme immortelle! (Ibid. : 178)

Georges guérit, retrouve sa fiancée, et la pièce se termine par une apothéose, tableau final coutumier du genre de la féerie, mais imprégné d'un ton très religieux. Le décor change aux derniers mots de Georges qui recouvre sa raison «en une sorte de cathédrale aérienne » :

Volsius joue alors une sorte d'Hosannah. La cathédrale se transforme de nouveau. $\mathrm{Au}$ fond, toute une «Gloire » resplendissante apparaît entourée d'anges. Ox luimême, vaincu par la sublimité de cette vision, s'incline à son tour.

Rideau (Ibid. : 180).

\section{Les arrière-plans du docteur $0 x$}

À part les allusions directes et indirectes aux Voyages extraordinaires, toute une série de références permettent de cerner le personnage du docteur Ox. Si dans la nouvelle citée de 1872, il soumettait toute la population d'une petite ville des Flandres à des expériences à base d'oxygène, il n'avait pas encore le caractère démoniaque qu'il revêt dans la pièce, il y est décrit comme "un étrange personnage, au sang chaud et impétueux, véritable excentrique échappé d'un volume d'Hoffmann» (Verne, 1874: 18) ${ }^{10}$. L'évocation de l'auteur romantique allemand n'est pas anodine car il fut une source d'inspiration du jeune Verne dès les années 1850. Le recueil de 1874 intitulé Le Docteur Ox réunit une partie des nouvelles écrites à cette époque et établit un curieux lien entre Hoffmann, l'œuvre de jeunesse d'une part, et le Voyage à travers l'impossible, de l'autre. 
Un hivernage dans les glaces, d'abord publié en 1855, relate une expédition dans les régions arctiques, qui préfigure le roman Voyages et aventures du capitaine Hatteras.

Dans Un drame dans les airs (à l'origine Un voyage en ballon, 1851), un aéronaute est harcelé par un fou qui le force à s'élever dans le ciel où il veut mourir en martyre de la science. Ses devises sont celles que proclamera à plusieurs reprises Georges Hatteras: "Se rapprocher de l'infini, c'est le comprendre ! (Ibid.: 158) et « Plus haut ! Plus haut ! (Ibid $., 168)$.

Maître Zacharius (le titre original de 1854 se poursuivit par ou l'horloger qui avait perdu son âme. Tradition genevoise) constitue un véritable hommage à Hoffmann, réunissant plusieurs aspects, thèmes et noms de son œuvre (Dehs, 2010). C'est l'histoire d'un horloger mégalomane qui perd sa foi en Dieu et est prêt à sacrifier sa famille à la science. Sa fille Gérande est poursuivie par le démon Pittonaccio (l'incarnation du temps et de la science blasphématoire) comme Éva le sera - aux mots près - par Ox une trentaine d'années plus tard. Zacharius est châtié au château d'Andernatt alors que Georges Hatteras, plus heureux, recouvrera sa raison au château d'Andernak - autant d'autoréférences entre les deux textes, comme Jules Verne aimait les multiplier dans ses œuvres.

Verne avait trouvé un modèle de ce procédé intertextuel dans la comédie Les Contes d'Hoffmann (1851) de Jules Barbier et Michel Carré avec lequel il collaborait, à cette époque, à plusieurs pièces de théâtre. C'est la même œuvre, reprise par Barbier seul, qui servit trente ans plus tard de livret à l'opéra portant le même titre de Jacques Offenbach (en 1876/77, il avait déjà mis en musique Le Docteur Ox de Jules Verne), montée à titre posthume le 10 février 1881 à l'Opéra-Comique de Paris. Aussi bien la comédie de 1851 que l'opéra de 1881 suivent la même structure, qui sera reprise par Verne et d'Ennery dans le Voyage à travers l'impossible, ainsi que le précise Jean-Michel Margot dans sa préface de la pièce :

Les Contes d'Hoffmann ont cinq actes: le prologue, le premier amour (Olympia, la poupée mécanique), le deuxième amour (Antonia, qui meurt en chantant), le troisième amour (Giuletta, qui vole l'ombre d'Hoffmann) et l'épilogue. La pièce de Verne et d'Ennery s'articule sur le même schéma, le prologue ouvrant l'acte premier et l'épilogue terminant le troisième acte de Voyage à travers l'impossible. Les deux héros, Hoffmann et Georges Hatteras, ont à choisir, le premier entre l'art et l'amour, le second entre la connaissance et l'amour (Verne, $2005: 12-13$ ).

Le docteur Ox du Voyage y trouve d'ailleurs ses modèles démoniaques dans les personnages du conseiller Lindorf, de Coppélius et du docteur Miracle. Les références à l'œuvre d'Hoffmann ont servi à Jules Verne pour se démarquer explicitement de l'idéologie vulgarisatrice prônée par la maison Hetzel, via un retour au « fantastique pur, que nulle raison physique ne peut accorder » (Verne sur Hoffmann : Verne, $1864: 194)$. Le retour au théâtre, cet amour déjà ancien et incessant, lui avait permis une liberté d'expression que son éditeur et ami Hetzel ne lui aurait jamais accordée. À une époque où la question laïque fut vivement débattue par la République (mais tranchée de manière définitive seulement après la mort de l'écrivain, en 1905) - une séparation entre l'Église et l'État que Verne rejeta à titre privé et, à partir de 1888, comme conseiller municipal de la ville d'Amiens - le Voyage à travers l'impossible constitua une évidente prise de position en faveur de la religion et contre une idéologie scientiste que Verne n'avait jamais adoptée.

Il y a un antécédent dans l'œuvre vernienne, au début de sa carrière d'écrivain, en 1863, lorsque Verne avait proposé à Hetzel, après Cinq semaines en ballon et le Capitaine Hatteras, la dystopie de Paris au $\mathrm{Xx}^{e}$ siècle. Ce roman porte en épigraphe une citation du journaliste 
Paul-Louis Courier, empruntée aux "Lettres au rédacteur du Censeur» ( $\left.\mathrm{n}^{\circ} \mathrm{IX}\right)$, qui s'oppose radicalement aux préoccupations relatives à l'instruction de la maison Hetzel :

O terrible influence de cette race qui ne sert ni Dieu, ni le roi, adonnée aux sciences mondaines, aux viles professions mécaniques! Engeance pernicieuse, que ne feraitelle pas si on la laissait faire, abandonnée sans frein à ce fatal esprit de connaître, d'inventer et de perfectionner ! (Courier, $1951: 36$ )

Ce que Courier avait écrit, dans un but satirique pour critiquer la censure réactionnaire de son époque, est pris à la lettre par Verne - l'éditeur Hetzel n'aurait pas pu publier un tel affront! Presque trente ans après, Voyage à travers l'impossible se range dans le même registre et souligne, par la multitude de ses allusions autoréférentielles et intertextuelles, l'unité des idées qui relie l'œuvre de jeunesse de Verne et les Voyages extraordinaires. Quelle que soit la valeur littéraire qu'on puisse accorder à cette pièce, le Voyage à travers l'impossible invite le public à relire les romans du cycle sous un angle insoupçonné.

\section{BIBLIOGRAPHIE}

COMPÈRE, Daniel (1991). Jules Verne écrivain. Genève : Droz.

Correspondance II (2001). Correspondance inédite de Jules Verne et de Pierre-Jules Hetzel (1863-1886).

Établie par Olivier Dumas, Piero Gondolo della Riva et Volker Dehs. Tome II (1875-1878). Genève : Slatkine.

Correspondance III (2002). Correspondance inédite de Jules Verne et de Pierre-Jules Hetzel (1863-1886). Établie par Olivier Dumas, Piero Gondolo della Riva et Volker Dehs. Tome III (1875-1886). Genève : Slatkine.

COURIER, Paul-Louis (1951). CEuvres complètes. Texte établi et annoté par Maurice Allem. Paris : Gallimard (Bibliothèque de la Pléiade, 59).

DEHS, Volker (2010). «Verne et Hoffmann », Revue Jules Verne (Amiens : Centre de documentation Jules Verne) n³1, pp. 10-26, avec les notes omises par inadvertance dans le $n^{\circ} 32,2011$, pp. 124-126.

DEHS, Volker (2011a). «Likao ou le Chinois éclipsé », Jules Verne \& $C^{i e}$. Bulletin du Club Verne, Amiens : Encrage, $\mathrm{n}^{\circ}$ 1, pp. 60-66.

DEHS, Volker (2011b). « Les drames du crépuscule. L'exemple de Famille-sans-nom », Revue Jules Verne (Amiens : Centre International Jules Verne) n 33/34, pp. 53-63.

HETZEL, Pierre Jules (1867). « Avertissement de l'éditeur », in Jules Verne, Voyages et aventures du capitaine Hatteras. Paris : Hetzel et $\mathrm{C}^{\text {ie }}$ (édition grand in- $8^{\circ}$ ), pp. I-II.

LAISsus, Joseph (1969). « Le voyage à travers l'impossible », Bulletin de la Société Jules Verne, $\mathrm{n}^{\circ} 12$, pp. 79-81.

LAROCHELLE, Paul (1960). Trois hommes de théâtre. Les trois Larochelle (1782-1930). Sans lieu : Éditions du Centre.

MARGOT, Jean Michel (2004). Jules Verne en son temps. Paris : Société d'édition Les Belles Lettres. 
STOULLIG, Édouard (1882). « Premières représentations », Le National n 5002, Lundi 27 novembre 1882 , p. 3.

VERNE, Jules (1864). «Edgard Poë et ses œuvres », Musée des familles, tome 31 ${ }^{\mathrm{e}}$, avril 1864, pp. 193-208.

VERNE, Jules (1874). Le Docteur Ox. Paris : J. Hetzel et C (édition in-18).

VERNE, Jules (1981). Voyage à travers l'impossible. Édité par François Raymond et Robert

Pourvoyeur. Paris : Jean-Jacques Pauvert.

VERNE, Jules (2005). Voyage à travers l'impossible. Édité par Agnès Marcetteau et Jean-Michel

Margot. Nantes : L'Atalante.

\section{NOTES}

1. Verne se rapporte à un roman d'Alphonse Brown qui venait de publier La Conquête de l'air (Paris : Glady frères) par un appareil plus lourd que l'air.

2. En préparation pour paraître dans le Bulletin de la Société Jules Verne.

3. Paul Larochelle est le fils d'Henry Larochelle, directeur du théâtre de la Gaîté-Lyrique où Kéraban fut monté. Gisette est le nom sous lequel Mme d'Ennery était connue alors qu'elle était encore actrice.

4. D'après les registres de la Société des auteurs et compositeurs dramatiques (Paris) pour le Théâtre de la Porte Saint-Martin, août 1879-décembre 1885.

5. Archives nationales, Paris. Dossiers de client de l'étude XCIII, MC/DC/XCIII/2. Document communiqué par Francis Marchand que je remercie de sa bienveillance.

6. Comme en témoigne Laissus (1969) qui, dans son article posthume, ne pouvait se baser que sur un article de la presse contemporaine.

7. Archives nationales, archives de la Censure, F/18/907. Le nom de Sigaux est cité par CharlesNoël Martin dans sa thèse inédite Recherches sur la nature, les origines et le traitement de la science dans l'œuvre de Jules Verne. Paris, Sorbonne, 1980, p. 611, note 449.

8. L'exemple le plus flagrant, mais non isolé, est le roman Le Rayon-Vert, de cette même année 1882, dans lequel Jules Verne oppose le peintre sympathique Olivier Sinclair au savant pédant Aristobulus Ursiclos.

9. Motif utilisé par Verne dans le dénouement original (mais resté inédit sur la pression d'Hetzel) d'Hector Servadac (1877) et repris dans le roman posthume La Chasse au météore (écrit en 1901, publié en 1986).

10. Je ne cite pas d'après la version originale (où ce passage se retrouve sans variantes), mais d'après le remaniement paru chez Hetzel en 1874.

\section{RÉSUMÉS}

Le texte de l'une des pièces les plus insolites de Jules Verne, le Voyage à travers l'impossible, est resté inédit pendant plus de cent ans. Après une seule mise en scène à Paris en 1882-83 et 97 représentations, cette pièce n'a connu qu'un succès mitigé et tomba vite dans l'oubli. Écrite en 
collaboration avec le dramaturge Adolphe d'Ennery, à mi-chemin entre le premier et le dernier des Voyages extraordinaires, elle constitue un singulier texte métatextuel s'en prenant, par son caractère purement fantaisiste, au genre rationnel des romans de la série et en recourant, par des allusions intertextuelles, à l'œuvre d'E.T.A. Hoffmann. Le présent article démontre comment Jules Verne, par un retour au genre dramatique et tout en évoquant quelques-uns de ses titres les plus célèbres, prend ses distances vis-à-vis de l'idéologie scientiste qui lui fut imposée par le programme de son éditeur Hetzel et intimement associée à l'auteur, dès son vivant, par ses lecteurs et l'opinion publique.

The text of a highly eccentric piece of Jules Verne, the Voyage à travers l'impossible, has remained unpublished for a hundred years. With 97 representations in Paris, the work had no remarkable success and was largely forgotten afterwards. Written in collaboration with the dramatist Adolphe d'Ennery, just at the same time as Verne was writing the Voyages extraordinaires, it constitutes a curious metatextual document which, for its fantastic character, calls in to question the seriousness of Verne's cycle of novels and draws on the works of German romantic E.T.A. Hoffmann. The article tends to show how Verne - by intertextual references to some of his most popular works - remains aloof of the scientist ideology claimed by his editor and which has been attributed to Verne during his lifetime by his readers.

\section{INDEX}

Keywords : fantastic play, Hoffmann (Ernst Theodor Amadeus), intertextuality, metatextuality, criticism of scientism

Mots-clés : fantastic play, Hoffmann (Ernst Theodor Amadeus), intertextualité, métatextualité, critique du scientisme

\section{AUTEUR}

\section{VOLKER DEHS}

Göttingen (Allemagne)

volker.dehs[at]web.de 